\title{
Increased percentage of Atrial Pace Ventricular Pace was associated with increased cardiovascular morality in patients with a DDD pacemaker
}

\section{Wen-Hsien Lee}

Kaohsiung Medical University

Chee-Siong Lee

Kaohsiung Medical University

Wei-Chung Tsai

Kaohsiung Medical University

Feng-Hsien Lin

Kaohsiung Municipal Siaogang Hospital

Wan-Ling Tsai

Kaohsiung Municipal Siaogang Hospital

Ying-Chih Chen

Kaohsiung Municipal Siaogang Hospital

Chang-Jen Chen

Kaohsiung Municipal Siaogang Hospital

Po-Chao Hsu

Kaohsiung Medical University

Chun-Yuan Chu

Kaohsiung Medical University

Tsung-Hsien Lin

Kaohsiung Medical University

Wen-Chol Voon

Kaohsiung Medical University

Ho-Ming Su ( $\square$ cobeshm@seed.net.tw)

Kaohsiung Medical University https://orcid.org/0000-0002-0858-2389

Research article

Keywords: pacemaker; cardiovascular mortality; total mortality

Posted Date: May 11th, 2020

DOI: https://doi.org/10.21203/rs.3.rs-25775/v1 
License: (c) (i) This work is licensed under a Creative Commons Attribution 4.0 International License. Read Full License 


\section{Abstract \\ Background}

Compared to single-chamber pacing system, dual chamber atrioventricular pacing system (DDD) was associated with reduced mortality and hospitalization for heart failure. High percentage of right ventricular pacing was reported to be associated with high cardiovascular events. However, another metaanalysis study showed ventricular pacing reduction modalities did not improve adverse clinical outcomes. Hence, the relationship between the pacing percentage and clinical outcomes is necessary to be further studied.

\section{Methods}

The present study was designed to evaluate the association of total and cardiovascular mortality with the percentages of atrial sense ventricular sense (ASVS), atrial sense ventricular pace (ASVP), atrial pace ventricular sense (APVS), and atrial pace ventricular pace (APVP) in patients with a DDD pacemaker. Study subjects were selected from patients arranged for permanent pacemaker follow-up at our special clinic. 177 patients with a DDD pacemaker were included. We collected their pacemaker follow-up data at their first visit for pacemaker follow-up after permanent pacemaker implantation.

\section{Results}

Among the 177 subjects, the mean follow-up to mortality was $2.44 \pm 1.03$ years. There were 22 and 10 patients documented as total and cardiovascular mortality. In the Cox proportional hazards regression analysis, old age $(P=0.030)$ and low hemoglobin $(P=0.007)$ were the predictors of increased total mortality and increased creatinine $(P=0.023)$ and high percentage of APVP (hazard ratio [HR], 1.030; 95\% confidence interval [Cl], 1.012-1.049; $\mathrm{P}=0.001$ ) were the predictors of increased cardiovascular mortality after multivariable analysis.

\section{Conclusion}

In patients with a DDD pacemaker, old age and low hemoglobin were associated with increased total mortality and high creatinene and high percentage of APVP were associated with increased cardiovascular mortality after multivariable analysis. Hence, reduction in unnecessary atrial and ventricular pacing in patients with a DDD pacemaker might be useful in improving cardiovascular prognosis.

\section{Background}


Cardiac pacing is the well-established treatment for patients with bradycardia. Pacemaker technology permits the use of either single-chamber ventricular pacemakers or dual-chamber pacemakers (DDD) for patients without permanent atrial fibrillation who require cardiac pacing. Compared to single-chamber pacing, dual-chamber pacing more closely resembles cardiac physiology by maintaining atrioventricular (AV) synchrony and thus reduces cardiovascular morbidity and mortality [1-3] and improves quality of life $[4,5]$.

Sakatani et al. found patients with a high percentage of ventricular pacing $(>90 \%)$ had a higher incidence of cardiac events than patients with a low percentage of ventricular pacing $(<10 \%)$ [6]. In contrast, Shurrab et al. concluded from a meta-analysis that reduction in unnecessary ventricular pacing failed to affect hard clinical outcomes in patients with preserved left ventricular function [7]. Hence, the relationship between the pacing percentage and clinical outcomes is necessary to be further studied. In the present study, we evaluated whether the pacing percentage in patients with DDD implantation was a useful parameter in prediction overall and cardiovascular mortality.

\section{Methods}

\section{Study Protocols and Design}

The patients with a DDD pacemaker implantation who visited the pacemaker follow-up clinic for a checkup in a regional hospital in southern Taiwan since April 2016. We consecutively enrolled all the patients at their first outpatient visit for regular pacemaker follow-up after their devices implantation. Exclusion were patients with malignancy. Finally, 177 patients were included in the present study. The study protocols were approved by the institutional review board committee of the Kaohsiung Medical University Hospital.

\section{Measurements of devices parameters during follow-up visit}

During the follow-up visit, we examined generator and leads status, including sensing, pacing threshold, impedance and percentage of sensing and pacing of atrial and ventricular leads. In particular, in order to decrease the percentage of ventricular pacing, we prolonged the PR interval to 20-30 ms longer than the intrinsic PR interval in patients with delayed AV conduction.

\section{Collection of basic characteristics, medical, and laboratory profiles}

Basic characteristics including age, gender, and comorbid conditions were acquired from medical records or interviews with our patients [8]. Blood samples were obtained within 3 month of enrollment. The diagnosis of diabetes mellitus and hypertension were defined by our previous study [8].

\section{Collection of mortality data}

Overall and cardiovascular mortality data were collected up to December 2019. All participants' mortality data were released from the Collaboration Center of Health Information Application, Ministry of Health and Welfare, Executive Yuan, Taiwan. 


\section{Statistical analysis}

Result data were presented as mean \pm standard deviation or percentage. Time to mortality events were modeled using the Cox proportional forward hazards models. All tests were 2-sided and their significance was defined as $P<0.05$. All statistical analyses were performed by SPSS 22.0 software (SPSS, Chicago, IL, USA).

\section{Results}

\section{Baseline characteristics among study patients}

The baseline characteristics of 177 study subjects are shown in Table 1 . The mean age was $72 \pm$ 14 years. The most common indication for pacemaker implantation was sick sinus syndrome, regarding $48 \%$ of the patients (81 cases). Advanced AV block, including Mobitz type II 2nd degree AV block, highdegree AV block, and 3rd degree AV block was the next in frequency, regarding $44 \%$ of the patients (78 cases). During pacemaker follow-up, the percentages of atrial sense ventricular sense (ASVS), atrial sense ventricular pace (ASVP), atrial pace ventricular sense (APVS), and atrial pace ventricular pace (APVP) were $32 \pm 37 \%, 33 \pm 40 \%, 24 \pm 33 \%$, and $11 \pm 20 \%$, respectively.

\section{Major predictors of overall and cardiovascular mortality in study patients}

The follow-up period to mortality was $2.4 \pm 1.0$ years in all patients. Mortality events were documented during the follow-up period, including total mortality $(n=22)$ and cardiovascular mortality $(n=10)$.

Table 2 shows the predictors of total mortality using Cox proportional hazards model. In the univariable analysis, increased total mortality was associated with increased age and decreased hemoglobin. After multivariable analysis, old age (hazard ratio [HR], 1.057; 95\% confidence interval [CI], 1.006-1.112; $\mathrm{P}=$ $0.030)$ and low hemoglobin $(\mathrm{HR}, 0.753 ; 95 \% \mathrm{Cl}, 0.612-0.927 ; \mathrm{P}=0.007)$ were still the predictors of increased total mortality. All of sensing and pacing percentages were not correlated to increased total mortality. In addition, the percentage of total right ventricular pacing (ASVP + APVP) was also not correlated to total mortality $(P=0.429)$.

Table 3 shows the predictors of cardiovascular mortality using Cox proportional hazards model. In the univariable analysis, increased cardiovascular mortality was associated with increased creatinine and high percentage of APVP. However, the percentage of total right ventricular pacing (ASVP + APVP) was not correlated to cardiovascular mortality $(P=0.182)$. After multivariable analysis, increased creatinine $(\mathrm{HR}, 1.385 ; 95 \% \mathrm{Cl}, 1.045-1.835 ; \mathrm{P}=0.023)$ and high percentage of APVP $(\mathrm{HR}, 1.030 ; 95 \% \mathrm{Cl}, 1.012-$ $1.049 ; \mathrm{P}=0.001$ ) were still the predictors of increased cardiovascular mortality.

\section{Discussion}


This study aimed to evaluate pacing percentage in predicting total and cardiovascular mortality in patients with a DDD pacmeaker implantation. We found old age and low hemoglobin were associated with increased total mortality, but all of sensing and pacing percentages were not associated with total mortality. In contrast, in addition to high creatinene, high percentage of APVP was significantly assoicated with increased cardiovascular mortality after multivariable analysis.

Compared to single-chamber ventricular pacemaker, DDD pacemaker has an important advantage because it more closely resembles cardiac physiology by maintaining AV synchrony. Hence, it might decrease cardiovascular morbidity and mortality, and may prolong survival and improve quality of life [4, 5]. Hence, in recent real-world practice, DDD pacemaker is a favor one unless patients have permanent atrial fibrillation. In patients with a DDD pacemaker, excessive right ventricular pacing may have an negative impact on cardiac function. Thus, it is necessary to optimize pacing parameters to mimize right ventricular pacing and maintain the cardiac function after pacemaker implant. In the study included patients with a DDD pacemaker by Zheng et al., subjects with a cumulative right ventricular percentage > $40 \%$ would have a more deterioation of cardiac function than those with a cumulative right ventricular percentage $\leq 40 \%$ [8]. In the study of Sakatani et al. included 268 consecutive patients with permanent pacemakers, they also found patients with a high percentage of ventricular pacing $(>90 \%)$ had a higher incidence of cardiac events than patients with a low percentage of ventricular pacing $(<10 \%)$ [6]. In contrast, Shurrab et al. demonstrated ventricular pacing reduction modalities did not improve clinical outcomes and were not superior to standard DDD programming in reducing incidence of permanent atrial fibrillation, all-cause hospitalization, or all-cause mortality [7]. In the present study, we similarly found the percentage of total right ventricular pacing (ASVP + APVP) was not correlated to total and cardiovascular mortaltiy.

APVP pacing mode has been considered inadequate in patients with a high degree of AV block and concomitant ischaemic heart disease. The possible explanation for this view was a fear of aggravating angina pectoris by a rate-dependent increase in myocardial oxygen consumption [9]. In addition, during ASVP pacing mode, both atria are activated via the intrinsic conduction system, but during APVP pacing mode, pacing of right atrial appendage resulted in a delayed electrical and mechanical activation of left atrium [10]. Hence, in the study of Bernheim et al., they found compared to ASVP pacing mode, APVP pacing mode could significantly worsen the myocardial performance index of left ventricle [11]. In the presen study, although the percentage of total right ventricular pacing was not a predictor of total and cardiovascular mortaltiy, the increased percentage of APVP was correlated to increased cardiovascular mortality. This finding implied that compared to ASVP pacing mode, APVP pacing mode might have a more deleterious impact on cardiovascular mortality. Therefore, reduction in unnecessary atrial and ventricular pacing in patients with a DDD pacemaker might be useful in improving cardiovascular prognosis.

\section{Study limitation}


There were several limitations in this study. First, the study generality was limited because our study patients were only included from one pacemaker follow-up clinic in a regional hospital in southern Taiwan. Second, our study was designed to assess the mortality events, so non-fatal events were not studied. Finally, the sample size of our study was relatively small, so the possibility of chance finding and the restricted power should be considered.

\section{Conclusions}

In patients with a DDD pacemaker, we found old age and low hemoglobin were associated with increased total mortality, but all of sensing and pacing percentages were not associated with total mortality. Besides, in addition to high creatinene, high percentage of APVP was significantly associated with increased cardiovascular mortality after multivariable analysis. Hence, reduction in unnecessary atrial and ventricular pacing in patients with a DDD pacemaker might be useful in improving cardiovascular prognosis.

\section{Abbreviations}

APVP, atrial pace ventricular pace; APVS, atrial pace ventricular sense; ASVP, atrial sense ventricular pace; ASVS, atrial sense ventricular sense; $A V$, atrioventricular; DDD, dual chamber atrioventricular pacing system; $\mathrm{Cl}$, confidence interval; $\mathrm{HR}$, hazard ratio.

\section{Declarations}

\section{Ethics approval and consent to participate}

The study protocols were approved by the institutional review board committee of the Kaohsiung Medical University Hospital. Written informed consent was obtained from all subjects.

\section{Consent for publication}

Not applicable

\section{Availability of data and material}

All data that support the present findings are included in our study. The datasets in the current study are available from the corresponding author on reasonable request.

\section{Competing interests}

The authors have declared no competing interest exists

\section{Funding}

Not applicable 


\section{Authors' contributions}

Conceptualization: Chee-Siong Lee, Ho-Ming Su

Data curation: Wen-Hsien Lee, Wei-Chung Tsai, Feng-Hsien Lin, Wan-Ling Tsai

Formal analysis: Wen-Hsien Lee, Wei-Chung Tsai, Wan-Ling Tsai, Ying-Chih Chen

Investigation: Wen-Hsien Lee, Ying-Chih Chen, Chun-Yuan Chu

Methodology: Chee-Siong Lee, Wei-Chung Tsai, Feng-Hsien Lin

Supervision: Chee-Siong Lee, Tsung-Hsien Lin, Wen-Chol Voon

Validation: Chee-Siong Lee, Feng-Hsien Lin, Chang-Jen Chen, Po-Chao Hsu

Writing - original draft: Ho-Ming Su

Writing - review and editing: Wen-Hsien Lee, Ho-Ming Su

All authors read and approved the final version of the manuscript

\section{Acknowledgements}

Mortality data were provided by the Collaboration Center of Health Information Application, Ministry of Health and Welfare, Executive Yuan.

\section{References}

1. Kosztin A, Boros AM, Merkel E, Schwertner WR, Behon A, Merkely B. Improved life expectancy in patients after dual-chamber pacemaker implantation. Kardiologia Polska. 2019;77(7-8):659-60.

2. Toff WD, Camm AJ, Skehan JD. Single-chamber versus dual-chamber pacing for high-grade atrioventricular block. N Engl J Med. 2005;353(2):145-55.

3. Connolly SJ, Kerr CR, Gent M, Roberts RS, Yusuf S, Gillis AM, Sami MH, Talajic M, Tang AS, Klein GJ, et al. Effects of physiologic pacing versus ventricular pacing on the risk of stroke and death due to cardiovascular causes. Canadian Trial of Physiologic Pacing Investigators. N Engl J Med. 2000;342(19):1385-91.

4. Lamas GA, Orav EJ, Stambler BS, Ellenbogen KA, Sgarbossa EB, Huang SK, Marinchak RA, Estes NA 3rd, Mitchell GF, Lieberman EH, et al. Quality of life and clinical outcomes in elderly patients treated with ventricular pacing as compared with dual-chamber pacing. Pacemaker Selection in the Elderly Investigators. N Engl J Med. 1998;338(16):1097-104.

5. Wong GC, Hadjis T. Single chamber ventricular compared with dual chamber pacing: a review. Can J Cardiol. 2002;18(3):301-7. 
6. Sakatani T, Sakamoto A, Kawamura K, Tanigaki T, Tsubakimoto Y, Isodono K, Kimura S, Matsuo A, Inoue K, Kitamura M, et al. Clinical Outcome After Permanent Pacemaker Implantation in Patients With a High Percentage of Ventricular Pacing. Int Heart J. 2015;56(6):622-5.

7. Shurrab M, Healey JS, Haj-Yahia S, Kaoutskaia A, Boriani G, Carrizo A, Botto G, Newman D, Padeletti $\mathrm{L}$, Connolly SJ, et al: Reduction in unnecessary ventricular pacing fails to affect hard clinical outcomes in patients with preserved left ventricular function: a meta-analysis. Europace: European pacing, arrhythmias, and cardiac electrophysiology : journal of the working groups on cardiac pacing, arrhythmias, and cardiac cellular electrophysiology of the European Society of Cardiology 2017, 19(2):282-288.

8. Lee WH, Hsu PC, Huang JC, Chen YC, Chen SC, Wu PY, Lee MK, Lee CS, Yen HW, Su HM. Association of Pulse Volume Recording at Ankle with Total and Cardiovascular Mortality in Hemodialysis Patients. Journal of clinical medicine 2019, 8(12).

9. Zheng L, Du X. Ventricular pacing on the prognosis of patients with pacemaker implantation. Cell Biochem Biophys. 2014;69(2):225-8.

10. Kristensson BE, Arnman K, Ryden L. Atrial synchronous ventricular pacing in ischaemic heart disease. European heart journal. 1983;4(9):668-73.

11. Cha YM, Nishimura RA, Hayes DL. Difference in mechanical atrioventricular delay between atrial sensing and atrial pacing modes in patients with hypertrophic and dilated cardiomyopathy: an electrical hemodynamic catheterization study. Journal of interventional cardiac electrophysiology: an international journal of arrhythmias pacing. 2002;6(2):133-40.

12. Bernheim A, Ammann P, Sticherling C, Burger P, Schaer B, Brunner-La Rocca HP, Eckstein J, Kiencke S, Kaiser $C$, Linka A, et al. Right atrial pacing impairs cardiac function during resynchronization therapy: acute effects of DDD pacing compared to VDD pacing. J Am Coll Cardiol. 2005;45(9):1482-7.

\section{Tables}

Table 1. Baseline characteristics in our study patients 


\begin{tabular}{lc}
\hline Characteristics & $\begin{array}{c}\text { All patients } \\
\text { (n = 177) }\end{array}$ \\
\hline Age (year) & $72 \pm 14$ \\
Male gender (\%) & 47 \\
Diabetes mellitus (\%) & 29 \\
Hypertension (\%) & 60 \\
Hemoglobin (g/dl) & $12.9 \pm 1.9$ \\
Creatinine (mg/dl) & $1.31 \pm 1.16$ \\
Total cholesterol (mg/dl) & $171 \pm 40$ \\
Triglyceride (mg/dl) & $109 \pm 55$ \\
Indication for PM implantation & \\
Advance AV block (\%) & 44 \\
Sick sinus syndrome (\%) & 46 \\
Others (\%) & 10 \\
Sensing and pacing percentage & \\
ASVS (\%) & $32 \pm 37$ \\
ASVP (\%) & $33 \pm 40$ \\
APVS (\%) & $24 \pm 33$ \\
APVP (\%) & $11 \pm 20$ \\
\hline
\end{tabular}

APVP: atrial pace ventricular pace; APVS: atrial pace ventricular sense; ASVP: atrial sense ventricular pace; ASVS: atrial sense ventricular sense; AV: atrioventricular; PM: pacemaker

Table 2. Predictors of total mortality using Cox proportional hazards model

\begin{tabular}{|c|c|c|c|c|}
\hline \multirow[t]{2}{*}{ meter } & \multicolumn{2}{|c|}{ Univariable analysis } & \multicolumn{2}{|c|}{ Multivariable analysis } \\
\hline & HR $(95 \% \mathrm{CI})$ & $\mathrm{P}$ & HR (95\% CI) & $\mathrm{P}$ \\
\hline (year) & $1.067(1.017-1.119)$ & 0.008 & $1.057(1.006-1.112)$ & 0.030 \\
\hline gender & $1.318(0.559-3.105)$ & 0.527 & & \\
\hline etes mellitus & $1.330(0.509-3.479)$ & 0.560 & & \\
\hline ərtension & $0.849(0.365-1.970)$ & 0.393 & & \\
\hline oglobin (g/dl) & $0.717(0.584-0.880)$ & 0.001 & $0.753(0.612-0.927)$ & 0.007 \\
\hline tinine (mg/dl) & $1.236(0.995-1.536)$ & 0.055 & & \\
\hline l cholesterol (mg/dl) & $1.001(0.988-1.014)$ & 0.917 & & \\
\hline yceride (mg/dl) & $0.992(0.980-1.004)$ & 0.178 & & \\
\hline \multicolumn{5}{|c|}{ :ation for PM implantation } \\
\hline lvance AV block & $0.759(0.314-1.832)$ & 0.540 & & \\
\hline ck sinus syndrome & $0.542(0.208-1.412)$ & 0.210 & & \\
\hline \multicolumn{5}{|c|}{ ing and pacing percentage } \\
\hline IS (\%) & $0.991(0.978-1.005)$ & 0.194 & & \\
\hline$J \mathrm{P}(\%)$ & $1.001(0.991-1.011)$ & 0.825 & & \\
\hline IS (\%) & $1.005(0.993-1.018)$ & 0.373 & & \\
\hline JP (\%) & $1.014(0.996-1.031)$ & 0.126 & & \\
\hline
\end{tabular}

HR, hazard ratio; CI, confidence interval; other abbreviations as in Table 1.

Table 3. Predictors of cardiovascular mortality using Cox proportional hazards model 


\begin{tabular}{|c|c|c|c|c|}
\hline \multirow[t]{2}{*}{ meter } & \multicolumn{2}{|c|}{ Univariable analysis } & \multicolumn{2}{|c|}{ Multivariable analysis } \\
\hline & HR $(95 \%$ CI) & $\mathrm{P}$ & HR $(95 \% \mathrm{CI})$ & $\mathrm{P}$ \\
\hline (year) & 1.049 (0.984-1.119) & 0.146 & & \\
\hline : gender & $0.980(0.263-3.653)$ & 0.976 & & \\
\hline etes mellitus & $1.423(0.364-5.566)$ & 0.610 & & \\
\hline srtension & $0.669(0.1925-2.330)$ & 0.525 & & \\
\hline oglobin $(\mathrm{g} / \mathrm{dl})$ & $0.800(0.590-1.086)$ & 0.149 & & \\
\hline tinine $(\mathrm{mg} / \mathrm{dl})$ & $1.352(1.047-1.745)$ & 0.021 & $1.385(1.045-1.835)$ & 0.023 \\
\hline l cholesterol $(\mathrm{mg} / \mathrm{dl})$ & $1.000(0.981-1.018)$ & 0.964 & & \\
\hline yceride (mg/dl) & $0.990(0.973-1.006)$ & 0.229 & & \\
\hline \multicolumn{5}{|c|}{ :ation for PM implantation } \\
\hline lvance AV block & $0.620(0.155-2.483)$ & 0.500 & & \\
\hline ck sinus syndrome & $0.360(0.075-1.736)$ & 0.203 & & \\
\hline \multicolumn{5}{|c|}{ ing and pacing percentage } \\
\hline IS (\%) & $0.996(0.978-1.014)$ & 0.633 & & \\
\hline$J \mathrm{P}(\%)$ & $0.999(0.984-1.015)$ & 0.942 & & \\
\hline IS (\%) & $0.986(0.960-1.012)$ & 0.282 & & \\
\hline JP (\%) & $1.032(1.012-1.052)$ & $<0.001$ & $1.030(1.012-1.049)$ & 0.001 \\
\hline
\end{tabular}

HR, hazard ratio; CI, confidence interval; other abbreviations as in Table 1. 